\title{
A PILOT STUDY ON VIDEO COLOR TRANSFER: A SURVEY OF USER-TYPE OPINIONS
}

\author{
Mairéad Grogan*, Emin Zerman*, Gareth W. Young* and Aljosa Smolic \\ $V$-SENSE, School of Computer Science, Trinity College Dublin, \\ College Green, Dublin 2, Dublin, Ireland
}

\begin{abstract}
Multimedia software products can be used to create and edit various aspects of online media. Recently, the affordances of mobile devices and high-speed mobile data networks mean that these editing capabilities are more readily available for mobile devices enabling a broader consumer-base. However, the precise role of the user in creative practice is often neglected in favor of reporting faster, more streamlined device functionality. In this paper, we seek to identify high-level human-computer interaction issues concerning video recoloring interfaces that are driven by the needs of different user-types via a methodological and explorative process. By conducting a pilot study, we have captured both quantitative and qualitative responses that formatively explore the role of the user in video recoloring tasks carried out on mobile devices. This research presents a variety of user responses to a video recoloring application, identifying areas of future investigation for explorative practices in user interface design for video recoloring visualization. These findings present important information for researchers exploring the use of state-of-art video recoloring processes and contribute to dialogues surrounding the study of mobile technology in use.
\end{abstract}

\section{KEYWORDS}

Color Transfer, User Study, Opinions, Usability

\section{INTRODUCTION}

In recent years, advances in mobile technology and the popularity of image and video sharing applications such as Instagram and Flickr have seen the platform for image and video editing tools shift from PCs to mobile phones. Editing tasks previously carried out by artists using software applications such as Adobe Photoshop and Premiere Pro are now achievable by casual creatives using simpler tools such as Instagram filters and Google Photo's in-app editing facilities. As these mobile editing tools rise in popularity, we must strive to understand the motivations and expectations of these new casual users when editing images and videos. For this purpose, a pilot study was undertaken to gain insight into how people with different levels of editing expertise use mobile applications when recoloring videos, assessing the users' interactions with the application interface, and exploring other measures such as user expectations and usability. In this paper, we outline the related work in this area (Sec. 2), describe the design of our recoloring application (Sec. 3), detail the methodology behind our user study (Sec. 4), present the results and data gathered (Sec. 5), and discuss these results (Sec. 6). Finally, the conclusions that can be drawn (Sec. 7).

\section{RELATED WORK}

One recoloring approach that has become very popular in recent years is palette-based image recoloring. These methods allow users to edit a small palette of colors to indicate what color changes they would like to see in their recolored images. Since these applications allow for more interactive experiences during the recoloring process, user studies are often undertaken to assess usability and quantify the overall user experience. In the work of Chang et al (2015), novice users were given an allotted time to recolor an input

\footnotetext{
* These authors contributed equally
} 
image to match a target image using different pieces of software. The difference between the recolored image and the target was then computed, along with the time taken to edit the image, and both were used to assess the usability of the applications. When assessing new tools, others compared the results generated by novice users using the proposed approaches to those generated by professional artists using tools available in Adobe Photoshop, such as the Replace Color tool, or image keying tools such as Keylight and Primatte (Aksoy et al., 2017, Chang et al., 2015, Zhang et al., 2017). These studies give an insight into how different pieces of software compare to professional tools for specific tasks, as well as providing an insight into the usability of these tools and whether the correct level of control is provided to recreate particular image results.

Another popular approach to image and video recoloring is example-based recoloring, where an example image provides the color palette used to recolor the input image (Arbelot et al., 2017, Ferradans et al., 2013, Grogan \& Dahyot, 2019, Reinhard et al., 2001) or video (Bonneel et al., 2013, Frigo et al., 2015, Grogan \& Dahyot, 2015) While the quality of example-based image recoloring approaches have been assessed subjectively and user studies implemented to determine which example-based method creates the most appealing results (Grogan \& Dahyot, 2019, Hristova et al., 2015), little research has considered how "usable" these methods are for different user-types, whether this type of approach allows users to create their desired results, whether more complex control is required by users, and what types of palette images should be provided. In terms of example-based video recoloring, many methods simply extend image techniques to video data by applying the color transfer function estimated for a single frame to all frames of the video (Frigo et al., 2015, Grogan \& Dahyot, 2015). While the quality of image recoloring techniques has been assessed through subjective experiments, their extensions to video data often have not (Grogan \& Dahyot, 2019).

With the continuous advances in mobile hardware, many state-of-the-art image editing tools have been transferred to mobile platforms, raising new questions about how such applications should be designed (Isenberg, 2016). These tools include image recoloring, neural style transfer and filtering methods (Ryffel et al., 2017, Dürschmid et al., 2017, Reimann et al., 2019, Semmo et al., 2017). Ryffel et al. extended their soft segmentation approach to an augmented reality application, allowing users to virtually recolor a select number of paintings in a museum (Ryffel et al., 2017). Paintings were decomposed into layers in a preprocessing step which meant that at runtime, paintings could be recolored and displayed on the user's mobile device in real time. A recent neural style transfer approach proposed using a network with a reduced number of layers to reduce computation time, relying on upsampling to create high resolution results from the low resolution network output (Semmo et al., 2017). Other neural style transfer mobile applications consider the usability of the application, devising methods to increase the learnability of the application through user driven redesigns (Reimann et al., 2019) and allowing users to manipulate different style parameters and presets, and even share styles between users (Dürschmid et al., 2017). In this paper, we propose a pilot study to formatively assess user interactions with an example-based mobile video recoloring application, and to explore potential differences in user opinion and usability in terms of both the recoloring interface and the video results.

\section{MOBILE COLOR TRANSFER APPLICATION}

In this paper, we consider the color transfer method proposed by Grogan et al. (Grogan \& Dahyot, 2015, 2019, Grogan et al., 2015). Proposed first by Grogan et al. (2015) and extended by Grogan \& Dahyot (2019), this method enables the color transfer of the source image to a targeted color distribution by selecting a palette image. The method uses an L2 based cost function and transforms the colors in the input image to that of the target image by estimating a thin plate spline transfer function $\varphi$, see Figure 1 . Although the original method was proposed for images, it is shown by Grogan \& Dahyot (2015) that applying the same transfer function which was estimated between a single frame of the source video and a target image, the method could also easily be extended to video. For this purpose, a single random frame can be selected from the source video, and the same $\varphi$ can be applied to all the frames of the said source video to avoid temporal inconsistencies. 

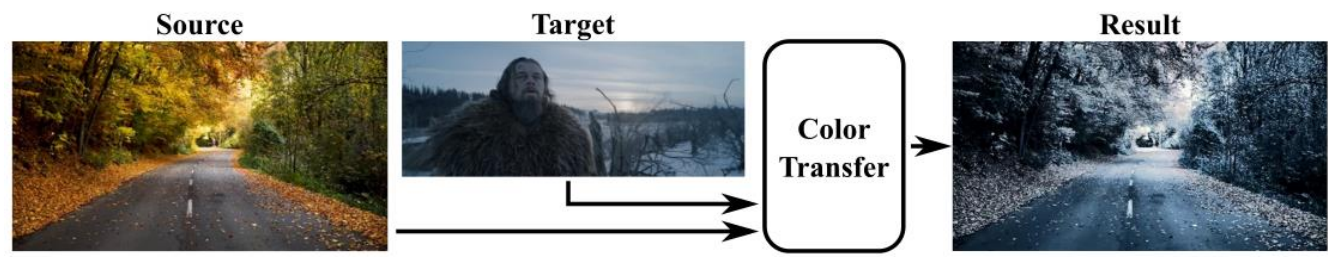

Figure 1. Example based color transfer used in this work

For this pilot study, the state-of-the-art video color transfer technology described was implemented by way of an Android application, see Figure 2. The application recolors video via the following steps. First, the user selects a source video from the phone gallery (Figure 2(a)) and a target color palette (Figure 2(b)) immediately after that. Although the application allows users to select a custom target image from the phone's gallery, in this study, the participants were asked to select from a predefined list ${ }^{\dagger}$ of palette images (Figure 2(c)) from famous movies that have a strong color feel, for example, The Revenant, 300, Alice Through the Looking Glass, etc. After users have selected both source video and target palette, they are brought to a preview screen that displays their selected video and target palette (Figure 2(d)). The color transfer process can then start after the user presses the 'Color Transfer' button. The resulting video is displayed after the color transfer process is completed (Figure 2(e)). On this last screen, users are given the option to download the resulting video.

The implemented application was a prototype and was designed as a front-end to back-end processing system residing on a remote server of a public university. In this framework, once users selected the input video and target palette, both video and palette were uploaded to the back-end where the color transfer function $(\varphi)$ was estimated and used to recolor the video. The recolored video was then sent back to the device and displayed to the user. The duration for uploading both source video and target palette to the server was 3.8 seconds on average for a video with $960 \times 540$ resolution. To accelerate the recoloring, source videos were resized to a resolution of $320 \times 180$ server-side to decrease processing times and reducing the download time for the result. The whole process took 8.6 seconds on average from pressing "Color Transfer" to the download of the resulting video.

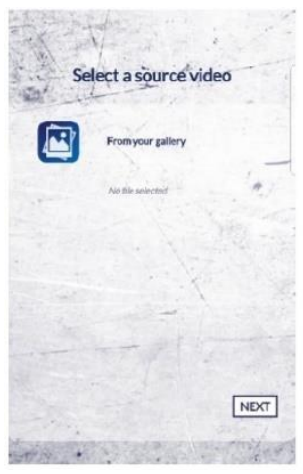

(a) Video selection

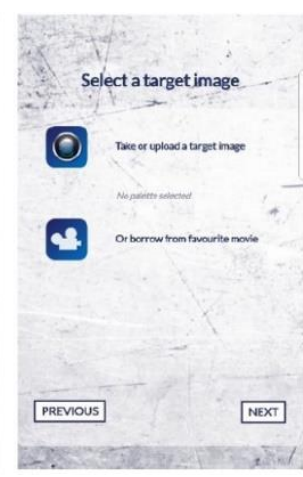

(b) Palette selection

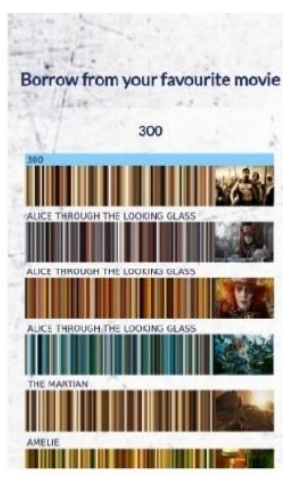

(c) Color palettes

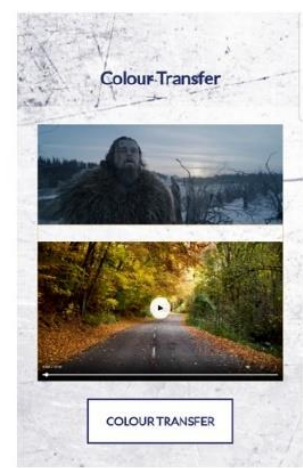

(d) Preview screen

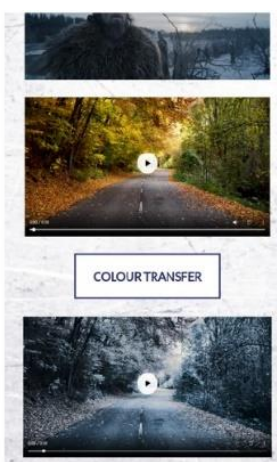

(e) Results screen

Figure 2. The user interface of the mobile color transfer application

\section{PILOT STUDY}

To collect data concerning the usability of the recoloring application a controlled pilot study was conducted. In this section, we discuss the methodologies applied. 


\subsection{Apparatus \& Setup}

A Huawei P9 Plus smartphone (with $1080 \times 1920$ display resolution) was used to run the Android application described previously. All participants used the same device under the same network conditions. Pretesting was undertaken to ensure that there were no network-related delays reported during the test. The study was conducted in a dedicated quiet and well-lit room with no windows. A total of 16 source videos and 12 target palettes $^{\dagger}$ were used in this study. Source videos were selected considering various features such as camera movement (tripod, drone, handheld), focus (all-in-focus, low depth of field), and theme (personal, professional, touristic). Target palettes were selected from famous movies in which the photography was color graded by industry professionals. Moreover, quantitative metrics such as color variation and spatial information were considered for the selection of source videos and target palettes. All source videos were of 10 seconds duration. This device set up ensured that we could identify which video and palette images were selected by each user for the reproducibility of results.

\subsection{Methodology}

Participant recruitment took place in the Republic of Ireland via an online call advertised in December 2019 on the mailing lists of the university and social media accounts of the research project, etc. Therefore, the cohort comprised of staff and students from the departments of engineering, computer science, and members of the university from outside of these disciplines. The study was then conducted within the latter part of January 2020. To avoid any bias which may have been caused by differences in device and location, the participants were invited to attend the laboratory at a time and date that suited their schedule. To preserve anonymity, participants were assigned a four-digit alphanumeric code. Following this, they were asked to report their age, gender, and occupation. To identify user-types within the cohort a user-cube approach (Nielsen, 1993) was implemented, therefore, participants were asked to self-identify their $(i)$ ability to use new technology, (ii) familiarity with the video and image editing domain, and (iii) expertise in the application and use of video and image editing applications on a fully labeled 5-point Likert scale.

To ensure all participants received the same instruction, they were presented with a scripted demonstration on how to use the mobile color transfer application. During this instruction, participants were shown each step of the color transfer procedure, and they viewed the resulting recolored video. After this, the test was conducted in two stages. In the first, participants were asked to complete at least two color transfer operations by themselves, selecting from pre-installed source videos and target palettes. During this time, participants were instructed to remain seated and hold the device at a comfortable distance. In the second stage of the study, the participants were asked to fill in a questionnaire. To collect user opinions related to the mobile recoloring application, this questionnaire included a Single Ease Question (SEQ) (Sauro \& Dumas, 2009), application-specific questions with fully labeled 5-point Likert scales, and open-ended answer options. Each session took no more than 30 minutes.

\subsection{Participants}

A total of 21 participants volunteered to take part in the study (Male $=14$; Female $=7$ ), with an average age of $31.5(M=31.50 ; S D=5.50)$. The cohort was comprised of students $(n=10)$, researchers $(n=9)$, and others $(\mathrm{n}=2)$. The participants self-reported that their ability to use new digital technology is "Good" to "Excellent" $(\mathrm{M}=4.29 ; \mathrm{SD}=1.01 ; \mathrm{CI}=0.43)$, their familiarity with the video and image recoloring domain is "Moderately Familiar" to "Very Familiar" $(\mathrm{M}=3.71 ; \mathrm{SD}=1.01 ; \mathrm{CI}=0.43)$, and their expertise in the use of video and image recoloring applications is "Average" $(\mathrm{M}=2.95 ; \mathrm{SD}=1.07 ; \mathrm{CI}=0.46)$. Using the three dimensions of technical ability, familiarity, and expertise, the participants were identified as different user-types. These user-types were novices (users with some simple experiences with the technology), primary and secondary end-users (users who routinely use this technology in their daily work activities or who are motivated by occasional, contextual, or intrinsic interests), and advanced users (tech-savvy professionals with a broad knowledge of related activities and tools, a high degree of competency, and comprehensive expertise). All participants were

\footnotetext{
${ }^{\dagger}$ For replicability, all the pre-selected source videos, target images, and their selection procedure are shared on our project webpage: https://v-sense.scss.tcd.ie/research/vfx-animation/mobile-video-color-transfer-project/.
} 
sufficiently versed in the use of digital technology to contribute meaningful discourse on the topic and the participant group could be considered as containing broad perspectives from novices to advanced users who were discreetly familiar with the recoloring domain. From this data, user-groups were logically formed as novices $(n=5)$, end-users $(n=9)$; and advanced users $(n=7)$, see Figure 3. The participant group size for a usability study remains a matter of debate; however, for studies related to problem discovery, a group size of 3-20 participants is typically valid, with 5-10 participants being a sensible baseline range (Macefield, 2009). Therefore, in the case of the presented study, the user-type groups were considered of large enough size for a pilot study to discover any severe problems in our video recoloring prototype design and not a direct user-type comparative study. Additionally, further limitations in the participant pool were found in gender type and employment sectors, constraining our extrapolation of results to the broader population.

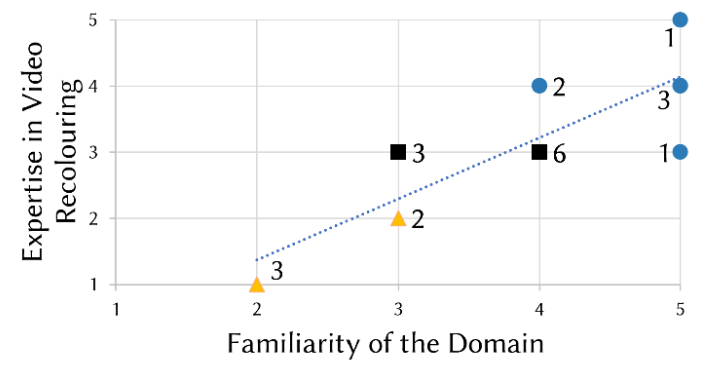

Figure 3. User-cube identifying user-groups: advanced (blue circles); end-users (black squares); novices (yellow triangles); numbers represent the number of users

\section{RESULTS}

Our pilot study sought to explore and identify elements of the video recoloring task that was of concern to our users. Both quantitative and qualitative data were collected, and areas of interest are reported below concerning the user interface. To begin, we assessed how difficult the recoloring task was to complete. The SEQ revealed that all users considered the application to be easy to use $(M=5.9, S D=1.31)$, see Figure 4(a). The results of a Kruskal-Wallis Test (a non-parametric method for comparing independent samples) indicated that there were no statistically significant differences in how difficult the user-types found the task, with $\mathrm{x}^{2}(2, \mathrm{n}=21)=0.49, \mathrm{p}=0.78$. Therefore, all users found the task "Easy" to complete $(\mathrm{Md}=6)$.

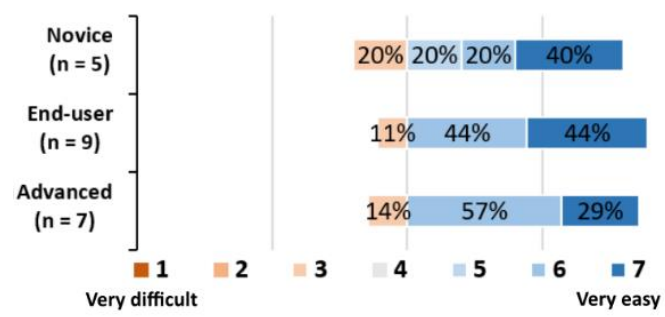

(a) The Single Ease Question (SEQ)

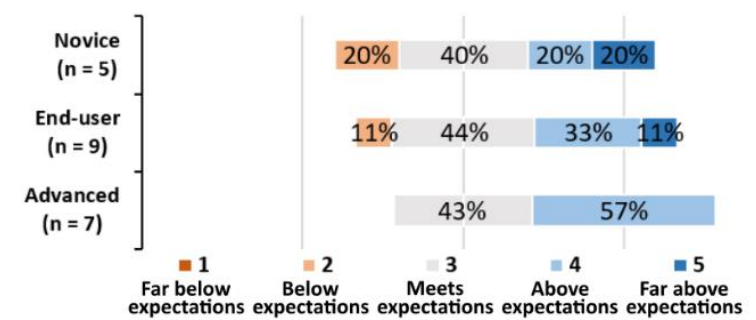

(b) User opinions about the number of palettes

Figure 4. Participant responses for SEQ and number of palettes

In response to the question "Were there enough palettes to get the results you wanted?" a median score of "Above Expectations" $(\mathrm{Md}=4)$ was measured. A Kruskal-Wallis test revealed no statistically significant differences in palette choices across the three different user groups, with $\mathrm{x} 2(2, \mathrm{n}=21)=0.248, \mathrm{p}=0.88$. When asked to expand upon their answers, users openly expressed that there were more than enough palettes provided for an exploratory task, with only two participants indicating that they would like more (a score of 2 ) and two others believing that the application gave more options far above their expectations (a score of 5). The advanced group recorded a median score of "Above expectations" $(\mathrm{Md}=4)$, and the novice and end-user groups both recorded median values of "Meets expectations" ( $\mathrm{Md}=3)$, see Figure 4(b). 
When asked "Which palette representation do you prefer?" from a selection of three choices, see Figure 5 , most participants preferred the combined representation of the movie frame and color palette (18/21). When asked to expand upon their preferences, users expressed their partialities for the movie still (2/21) as it was "simple", and felt that "[the palette] doesn't mean much to me" - KI74 (novice) and that the movie frame "gives a good example of the color palette" - MC75 (end-user). Statements for the color palette alone $(1 / 21)$ highlighted that there was "no bias in terms of content." - ZA60 (advanced user). With the color palette and movie still combined, the participants were able to understand how the image and colors related to the recolored video output, giving them a better understanding of how the color transformation would look.

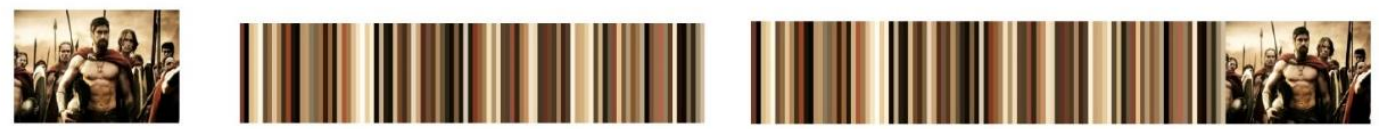

(a) Movie frame only

(b) Color distribution only

(c) Movie frame and color distribution combined

Figure 5. Palette representation

Participants were also asked "Would you prefer to use a recoloring application that provides color palettes or one that uses example images of the filters? Please explain why.", referring to Figure 6(a). Most participants preferred having example images as they seemed "more intuitive" and expressed that users "can directly evaluate the result". Moreover, the examples images were said to be helpful for users "to contextualize and visualize the result" - HM41 (advanced user). Some of the participants expressed that they liked the color palette representations in the application as " $[\mathrm{I}]$ prefer color palettes, because filters don't give much idea about the outcome, and they may not match our expectations" - IS42 (novice). Similarly, as mentioned by one advanced user, their preference was dependent on what they wanted to achieve: "When I know what look I want, color palettes. When I don't know what look I want, then example images" - CT77 (advanced user). Many of the participants would have liked to have seen example images when recoloring was taking place so they could get a better idea of what the output would look like. It was felt that it would be easier to directly evaluate the result and would be more intuitive to use.

To compare different types of interaction possibilities, the participants were asked "Do you think it would be easier to use this recoloring application rather than other types of existing filters? Please explain why.", see Figure 6(a). Participants' responses were mixed and not distinctly indicative when comparing user-type profiles. Many participants were in favor of the current recoloring application, while other members of the cohort were in favor of using existing user interfaces from familiar recoloring systems. Some participants also expressed that their opinions would be influenced by specific task factors, while other participants said that there were no distinguishing differences between the current recoloring application and other recoloring filter user interfaces. From the analysis of the qualitative user feedback, those in favor of the recoloring application thought that the current recoloring application was "less complicated", or it made "results more unique". One participant commented: "this recoloring application does most of the work for you if you want to quickly change the mood of an image/video" - BG78 (novice). Participants who were in favor of other, existing filter user interfaces said that they liked the instant feedback and "a preview down below" these interfaces provided "to give a quick idea of what the result might look like" - KI74 (novice). If provided with instant feedback, users "can test different filters very easily" - XC97 (advanced user). Users' opinions that were based purely upon the circumstances of the experiment stated that "if I want to change the mood, I will use the recoloring app. If the change I want is very small, maybe I would prefer a filter." - BV46 (end-user). Regardless of their personal preferences, many participants indicated that it was important to be able to control the effects and to tweak parameters, as "I often change the parameters of the filters when I do use them" - SQ34 (end-user). 

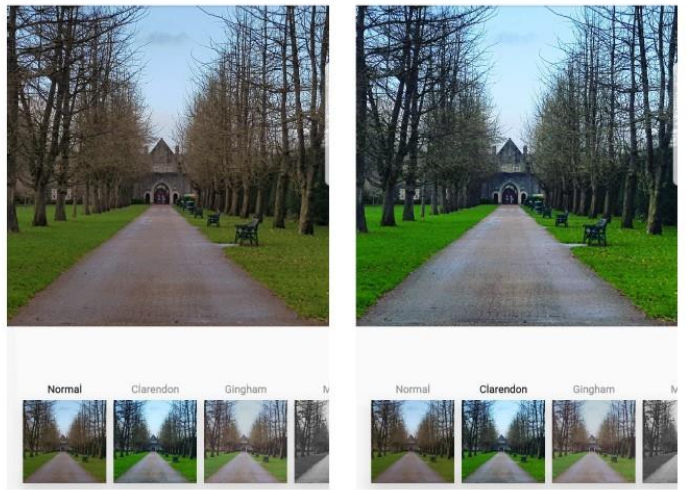

(a) Instagram filters

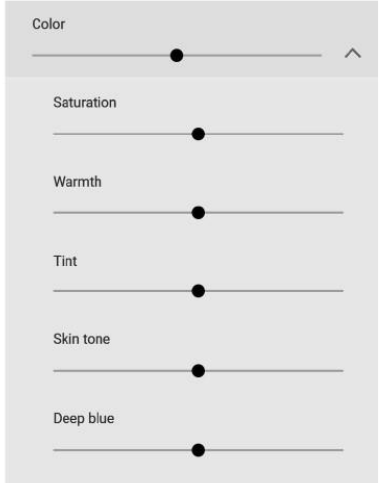

(b) Complex controls

Figure 6. Visual representations of different image and video editing tools

The participants were asked "Would you like more complex control over the recoloring application to create more appealing or custom looking videos? Please explain why.", referring to Figure 6(b). More than half of the participants declared that they would like more complex controls over those provided in the current recoloring application. Most of the participants stated that it would be great to "tweak the results" and to "correct errors" for "refinement" after initial results were processed, as "the application provides a great initial result but with refinement, it could be even better" - HM41 (advanced user). Although being more positive, some users suggested that "otherwise it does not add much to preset filters" - SK56 (end-user) or "it's good to have the option. However, I probably wouldn't use the advanced controls often!" - XC97 (advanced user). One user believed that it could be useful "if I were using it with professional software, but not for a social media app" - WD79 (advanced user). Participants that gave negative responses declared that they either "prefer a simple interface" or that it currently is "[a] little too complicated". The remainder of the participants were indecisive. One novice user acknowledged that "it might be nice to have in the rare case I want fine adjustment, but I rarely use these controls or keep the results from using them" - KI74 (novice). On the other hand, participants also wanted to have access to both options as "I will have a simple version, and for advance users let them customize it" - BV46 (end-user).

When asked "Do you think that a color palette is easier to use than the more traditional image and video editing tools that offer more custom color change options? Please explain why.", referring to Figure 6(b), participants mostly agreed that the recoloring application was easier to use but that this comes at a trade-off since they also have less control. These participants expressed that it was "more intuitive" and "it's easier to imagine the results without being an expert" - GS24 (end-user), while some users said that the current application was "easier because there are fewer parameters to tune" - MU69 (novice). It was also suggested that "it is less effort but leaves you feeling you don't have full control over the results" - YG89 (end-user). Participants also commented that the "feel" of the video was easier to change with the recoloring application, as "choosing a palette is more like choosing a mood" - JN33 (end-user). Some participants were indecisive on how difficult the current recoloring application was and answered the question without giving any explicitly positive or negative responses. These participants balanced how the recoloring results would change concerning the user's level of expertise and experience. For example, one end-user noted that "the color palette is quick and easy for social media use, but I still prefer full control for professional use" - HL75.

In the last section of the questionnaire, participants were asked to provide their opinions and suggestions regarding the ease of use of the user interface or discuss possible improvements that could be made. In general, advanced users were more critical of the layout and the technical limitations that they presented. On the other hand, participants who identified as end-users and novices suggested more practical changes, for example, changes in the color palettes or visualizations of the selected video and palette combination. Furthermore, positive comments regarding the application - "impressive" and "easy to use" - were also from this cohort. The three main areas of improvement were identified from participant comments on layout, technical limitations, and ease of use. 


\section{DISCUSSION}

This Android-based, mobile phone video recoloring application was designed as a prototype, and as such, the questions asked of the participants were aimed at highlighting possible research directions and improvements in the initial user interface design. The SEQ demonstrated how the mobile color transfer application was easy to use by all user-types. The task was clear and straightforward for novices, encouraged end-users to try new things, and proved adequate for most advanced users' needs. Furthermore, the user interface was considered easy to use when compared to other filter interfaces too. Participants' responses to other areas of the user interface were mixed regardless of the user-types and were often described as being task-dependent. Although the color transfer application had fewer parameters to tune and gave "a unique film look", the example images provided by filters gave instant feedback, which was sought after by users. The results of the study also indicated that the number of palettes provided either met expectations (for novices and end-users) or was above expectations (for advanced users). This suggests that 12 different target palettes would be enough for a mobile recoloring application. Moreover, participants preferred the combined palette representation, stating that they could understand how the color transfer would take place since the combined representation showed both the dominant colors and how they might be distributed in the recolored video. Regarding complex controls, participants noted that it would be good to have the option to tweak the results although these complex controls would probably not be used often. This shows the need for a simple interface for novice users and an expanded user interface option to satisfy end-users or advanced users. Regarding the interaction, most users preferred the example images that filters provided as it allowed the user to quickly evaluate the recolored result compared to the current recoloring application. Nevertheless, they also preferred the current recoloring application over the more traditional image and video editing tools, as the application was found easier to use. This shows that using simple interfaces that provide fast feedback is appealing to most of the users and user-types.

\section{CONCLUSION}

In this paper, we report on a pilot study that identifies human-computer interaction issues related to a state-of-the-art video recoloring application. For three different user-types, we formatively explore key aspects of the video recoloring application related to usability, design choices, and user-type expectations from such a mobile application. We found that the color transfer application was easy to use by all user-types. Moreover, the different user-types also agreed that the number of palettes and the palette representation met their expectations. While praising its simplicity, the users also requested additional features such as optional complex controls and instant feedback options. The results show that there are some qualitative differences among user-types. The novices we engaged with scored more broadly when compared to the other user-types, which can be analyzed in future work relating to motivations for knowledge building and advanced user interactions.

We believe that these results can help developers and researchers expand existing dialogues to improve user experiences and create new user interfaces for video recoloring applications. Particularly, we believe that user-types vary in their motivations, expectations, satisfaction, and perceived usability of mobile applications, as various factors impact their confidence when concerning their previous knowledge and experiences with video editing software. Therefore, future application developments and usability studies will focus on the addition of advanced "accelerator" options for more complex recoloring control, the development of sample-based feedback in the form of cell images and palettes, and explore the perceived usability and users' experiences of existing user interfaces (e.g. Instagram, Google Photos, etc.) that take different approaches to these issues. 


\section{REFERENCES}

Aksoy, Yagiz et al, 2017. Unmixing-based soft color segmentation for image manipulation. ACM Transactions on Graphics, Vol. 36, No. 2, pp. 19:1-19:19.

Arbelot, Benoit et al, 2017. Local texture-based color transfer and colorization. Computers \& Graphics, Vol. 62.

Bonneel, Nicolas et al, 2013. Example-based video color grading, ACM Transactions on Graphics, Vol. 32, No. 4.

Chang, Huiwen et al, 2015. Palette-based photo recoloring. ACM Transactions on Graphics, Vol. 34, No. 4.

Dürschmid, Tobias et al, 2017. ProsumerFX: Mobile Design of Image Stylization Components. Proceedings of SIGGRAPH Asia 2017 Mobile Graphics \& Interactive Applications, Bangkok, Thailand.

Ferradans, Sira et al, 2013. Regularized discrete optimal transport. In: Kuijper, Arjan, Bredies, Kristian, Pock, Thomas, \& Bischof, Horst eds, Scale Space and Variational Methods in Computer Vision, Berlin: Springer Berlin, pp. 428-439.

Frigo, Oriel et al, 2015. Optimal transportation for example-guided color transfer. In: Cremers, Daniel, Reid, Ian, Saito, Hideo, \& Yang, Ming-Hsuan eds, Computer Vision - ACCV 2014, Cham: Springer, pp. 655-670.

Grogan, Mairéad \& Dahyot, Rozenn, 2015. L2 registration for colour transfer in videos. Proceedings of the $12^{\text {th }}$ European Conference on Visual Media Production (CVMP), London, UK.

Grogan, Mairéad \& Dahyot, Rozenn, 2019. L2 divergence for robust colour transfer. Computer Vision and Image Understanding Vol. 181, pp. 39-49.

Grogan, Mairéad et al, 2015. L2 registration for colour transfer. 23rd European Signal Processing Conference (EUSIPCO). Nice, France, pp. 1-5.

Hristova, Hristina et al, 2015. Style-aware robust color transfer. Proceedings of the Workshop on Computational Aesthetics (CAE), Goslar, Germany, pp. 67-77.

Isenberg, Tobias, 2016. Interactive NPAR: What type of tools should we create? Proceedings of the Joint Symposium on Computational Aesthetics and Sketch Based Interfaces and Modeling and Non-Photorealistic Animation and Rendering, Lisbon, Portugal, pp. 89-96.

Macefield, Rich, 2009. How to specify the participant group size for usability studies: a practitioner's guide. Journal of Usability Studies, 5(1), 34-45.

Nielsen, Jakob, 1993. Usability engineering. Academic Press, Boston.

Pitié, François et al, 2005. N-dimensional probability density function transfer and its application to color transfer. Tenth International Conference on Computer Vision (ICCV), Beijing, China, pp. 1434-1439.

Reimann, Max et al, 2019. Locally controllable neural style transfer on mobile devices. The Visual Computer Vol 35, pp. 1531-1547.

Reinhard, Erik et al, 2001. Color transfer between images. IEEE Computer Graphics and Applications, Vol. 21, No. 5.

Ryffel, Mattia et al, 2017. AR Museum: A mobile augmented reality application for interactive painting recoloring. In Multi Conference on Computer Science and Information Systems 2017, Lisbon, Portugal.

Sauro, Jeff \& Dumas, Joseph S., 2009. Comparison of three one-question, post-task usability questionnaires. Proceedings of the SIGCHI conference on human factors in computing systems, pp. 1599-1608.

Semmo, Amir et al, 2017. Pictory: Combining Neural Style Transfer and Image Filtering. Proceedings of ACM SIGGRAPH 2017 Appy Hour, Los Angeles, California.

Zhang, Qing et al, 2017. Palette-based image recoloring using color decomposition optimization. IEEE Transactions on Image Processing, Vol. 26, No. 4, pp. 1952-1964. 